\title{
Disciplines scolaires et construction identitaire
}

\section{Nicole Biagioli et Jean-Jacques Legendre}

\section{(2) OpenEdition}

Journals

Édition électronique

URL : http://journals.openedition.org/trema/2522

DOI : 10.4000/trema.2522

ISSN : 2107-0997

\section{Éditeur}

Faculté d'Éducation de l'université de Montpellier

\section{Édition imprimée}

Date de publication : 1 décembre 2010

Pagination : 23- 33

ISSN : 1167-315X

\section{Référence électronique}

Nicole Biagioli et Jean-Jacques Legendre, «Disciplines scolaires et construction identitaire », Tréma [En ligne], 33 - 34 | 2010, mis en ligne le 01 décembre 2012, consulté le 19 avril 2019. URL : http:// journals.openedition.org/trema/2522 ; DOI : 10.4000/trema.2522

Ce document a été généré automatiquement le 19 avril 2019

Trema 


\title{
Disciplines scolaires et construction identitaire
}

\author{
Nicole Biagioli et Jean-Jacques Legendre
}

1 Afin de rester accessible, le savoir humain, toujours plus complexe, nécessite des restructurations d'autant plus indispensables que son institutionnalisation s'accroît sous l'effet de la démocratisation de l'enseignement et se diversifie par la participation croissante de l'ensemble des organismes sociaux, école mais aussi media, finance, industrie, etc. à son développement. Il s'agit désormais d'introduire la formation à la mixité scolaire dans le processus de masterisation et de professionnalisation.

2 En balisant les savoirs et en adaptant leur évolution à celle de la société, les disciplines scolaires préservent l'accès à la connaissance pour tous. Leur identification et l'analyse de la perception de leurs relations est l'objectif de la présente étude qui se situe dans le cadre des recherches menées par l'équipe pluridisciplinaire I3DL, Interdidactique, didactique des disciplines et des langues, de l'université de Nice à laquelle nous appartenons, et qui étudie les phénomènes résultant de la coexistence des disciplines dans le socle commun.

3 Les données expérimentales ont été recueillies à l'aide de l'enquête internationale électronique en six langues Harry Potter et le système éducatif. Passer par un intermédiaire romanesque nous a permis de rendre le questionnaire attractif afin d'inciter le maximum de personnes (9325 au 5 octobre 2009) à répondre à ses 180 questions, mais aussi de récolter des réponses sincères et aussi indépendantes que possible des discours dominants. La série Harry Potter a été sélectionnée parce qu'elle associe les sphères privée et scolaire. En outre, son succès mondial donne accès à un lectorat nombreux, motivé et très diversifié sur les plans géographique, politique, linguistique et culturel. 


\section{Disciplines favorites - Disciplines détestées}

4 Afin d'apprécier la manière dont les disciplines sont perçues par les apprenants, nous avons analysé les réponses, arrêtées au 20 juin 2009, à neuf questions, quatre sur les disciplines et cinq sur les qualités souhaitées chez ceux qui les enseignent.

5 Le traitement des réponses a été réalisé selon trois axes: analyse des deux tableaux à plat présentant les choix disciplinaires, croisement des deux variables fermées qui révèle les corrélations entre choix ; analyse des réponses aux questions ouvertes "Pourquoi?" qui donne accès aux justifications des enquêtés.

6 Comparons avec les tableaux $1 \mathrm{~A}$ et $1 \mathrm{~B}$ les pourcentages des réponses sur la matière préférées et sur la matière la moins aimée.

\begin{tabular}{|c|c|c|c|}
\hline Natère préférée $1 \mathrm{~A}$ & $\% \mathrm{cot}$ & Matière la moins amée $1 \mathrm{~B}$ & $\%$ ot \\
\hline Histoire & $18,2 \%$ & Mathematiques & $21,6 \%$ \\
\hline Langues étrangères & $15,2 \%$ & Education physique et sportive & $11,4 \%$ \\
\hline Mathematiques & $14,0 \%$ & Philosophie & $10,2 \%$ \\
\hline Sciences de la vie et de la terre (SVT) & $11,1 \%$ & Physique & $9,8 \%$ \\
\hline LS & $10,9 \%$ & Technologie & $5,7 \%$ \\
\hline Arts plastiques (dessin, peinture, sculpture) & $6,6 \%$ & Arts plastiques (dessin, peinture, sculpture) & $5,6 \%$ \\
\hline Chimie & $4,6 \%$ & Sciences teonomiques et sociales & $5,2 \%$ \\
\hline Physique & $3,4 \%$ & Langues anciennes (grec ancien, latin) & $5,0 \%$ \\
\hline Education physique of sportive & $3,4 \%$ & Musique et chant & $4,8 \%$ \\
\hline Philosophie & $3,1 \%$ & Histoire & $4,5 \%$ \\
\hline Musique et chant & $2,8 \%$ & Langues etrangeres & $4,0 \%$ \\
\hline Sciences économiques et sociales & $2,0 \%$ & LS & $3,5 \%$ \\
\hline Technologie & $1,7 \%$ & Chimie & $3,2 \%$ \\
\hline Langues anciennes (grec ancien, latin) & $1,6 \%$ & Geographie & $2,9 \%$ \\
\hline Géographie & $1,4 \%$ & Sciences de la vie of de la terre (SVT) & $2,5 \%$ \\
\hline Total & $100,0 \%$ & Total & $100,0 \%$ \\
\hline
\end{tabular}

7 Une discipline fait débat : les mathématiques, matière préférée d'un groupe important (14\%) et matière la moins appréciée par un groupe non moins important (22\%). Cet antagonisme est à rapprocher de la division des disciplines en familles qui apparaîtra lors du croisement des deux variables fermées.

8 A l'opposé, la géographie est une discipline "transparente", placée en fin de liste à la fois des disciplines préférées et des disciplines les moins aimées. Ni aimée, ni détestée, elle ne soulève pas les passions.

9 La division de l'échantillon en sous-groupes liés à la langue de scolarisation (LS) : France, Allemagne + Autriche (D + A), Etats-Unis + Grande Bretagne (USA + GB), Canada français, a permis de constater l'existence de nettes différences entre les choix disciplinaires des individus appartenant à des sous-groupes LS différents. Le tableau 2 présente les pourcentages de citations de chaque discipline comme discipline favorite dans les quatre sous-groupes. 
obtient des images des différentes identités culturelles dans leurs composantes scolaires dont certaines s'avèrent très dépendantes de l'environnement géopolitique.

Le groupe germanique, contrairement aux autres groupes, ne classe pas l'histoire parmi ses disciplines favorites. L'existence d'un passé culpabilisant peut éclairer ce phénomène.

11 Tableau 2: Comparaison des matières préférées dans les quatre sous-groupes. Les pourcentages en caractère gras correspondent aux trois disciplines les plus appréciées

\begin{tabular}{|c|c|c|c|c|}
\hline & $F$ & $D+A$ & USA+G B & Canada francais \\
\hline Histoir $\mathrm{e}$ & 18,2 & 10,9 & $\mathbf{1 3 , 0}$ & $\mathbf{1 8 , 5}$ \\
\hline Langues étrangeres & 15,2 & $\mathbf{1 8 , 4}$ & 7,0 & 6,7 \\
\hline Mathématiques & 14,0 & 13,3 & 7,3 & 11,3 \\
\hline $\begin{array}{c}\text { Langue du pays } \\
\text { de scolar isation }\end{array}$ & 10,9 & 11,4 & 26,2 & 14,9 \\
\hline SVT & 11,1 & 7,2 & 7,3 & 2,6 \\
\hline Arts plastiques & 6,6 & 10,4 & 16,6 & 16,9 \\
\hline EPS & 3,4 & 10,4 & 2,0 & 5,6 \\
\hline Chimie & 4,6 & 4,0 & 3,0 & 10,3 \\
\hline Musique , chant & 2,8 & 6,0 & 6,3 & 0,5 \\
\hline Physique & 3,4 & 6,0 & 0,7 & 0,5 \\
\hline Philosophie & 3,1 & 1,2 & 2,7 & 2,1 \\
\hline SES & 2,0 & 1,9 & 2,3 & 2,6 \\
\hline Gégraphie & 1,4 & 3,0 & 0,7 & 2,1 \\
\hline Technologie & 1,7 & 1,1 & 3,0 & 0,0 \\
\hline Langues anc & 1,6 & 1,2 & 2,0 & \\
\hline
\end{tabular}

Les Anglo-saxons, quant à eux, rejettent les langues étrangères et plébiscitent leur langue, alors que les autres apprécient plus les langues étrangères que les leurs, ce qui peut s'expliquer par la suprématie actuelle de l'anglais au plan mondial.

On notera que les trois disciplines favorites du groupe des Canadiens français sont les mêmes que celles du groupe anglo-saxon. Ce choix se différencie nettement de celui des Français. Il révèle la fragilité de leur revendication identitaire, tout au moins dans le domaine de la culture scolaire.

Est remarquable aussi l'homogénéité de l'échantillon "français" pour le choix de la discipline favorite selon la profession du père ${ }^{1}$. En effet, les Français de chacune des huit catégories socioprofessionnelles envisagées ont classé une seule discipline, l'histoire, comme matière favorite. Pour l'Allemagne et l'Autriche, ce choix se porte sur quatre matières : l'histoire, les mathématiques, les langues vivantes et les arts plastiques. Pour les Anglo-saxons, sur l'histoire, l'anglais, les mathématiques et les arts plastiques. On mesure ainsi sans doute les effets unificateurs du centralisme du système éducatif français qui, en normalisant les programmes, normalise les représentations des disciplines. A l'inverse, les systèmes éducatifs plus décentralisés des pays anglo-saxons ou germaniques produisent des représentations plus variées.

La recherche des relations existant entre les représentations que les élèves ont des différentes disciplines nous a conduit à réaliser une Analyse Factorielle des 
Correspondances sur 7366 observations en croisant les réponses aux deux questions fermées. Les résultats obtenus sont présentés de manière synthétique à la figure 1 .

On y remarque tout d'abord la répartition des disciplines autour d'un axe vertical en deux familles : à gauche les disciplines relevant des sciences humaines, à droite celles relevant des sciences exactes. Cette tendance est encore plus accentuée si l'on prend le seul échantillon français (non représenté ici), qui est aussi le seul à user du stéréotype oppositif scientifique/littéraire traité infra. On pourrait y voir une preuve de l'influence du système des sciences élaboré par A. Comte ${ }^{2}$ sur l'organisation des études en France.

La position atypique de l'EPS à la frontière entre sciences de l'homme et sciences de la nature corrobore les préoccupations des observateurs à son endroit ${ }^{3}$. Un second axe horizontal sépare les disciplines tournées vers la matière, en haut, de celles tournées vers les signes, en bas.

Figure 1: Carte des disciplines : La comélation des choix entre deux discipines dépend du produi t scalaire des deux vecteurs joignant lorigine et les spots associés

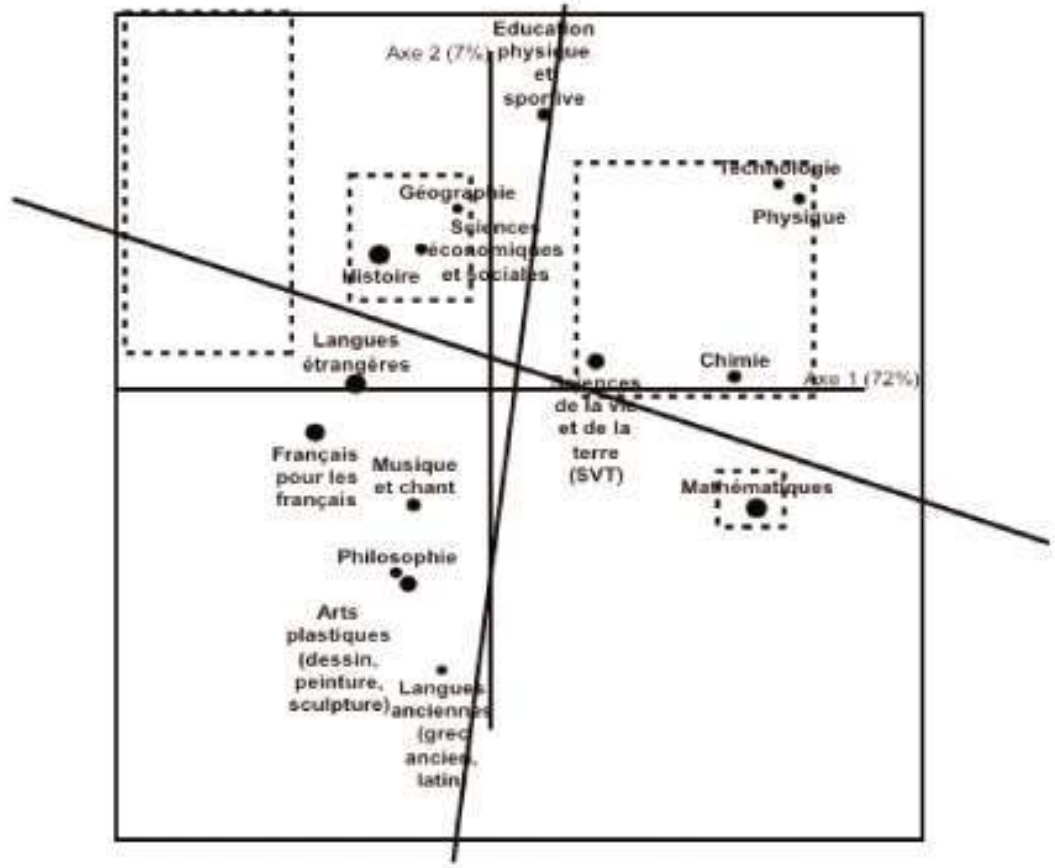

17 La proximité des spots associés, plus grande à droite qu'à gauche, indique que la représentation des relations entre sciences exactes est plus cohérente que celle des relations entre sciences humaines. La famille scientifique la plus récente semble n'avoir pas encore réussi à rendre sa structure lisible au grand public, même au travers de la transposition scolaire. Celle-ci semble plus proche du positivisme scientiste du XIXème siècle que de l'actualité scientifique du XXIème.

\section{II- Disciplines et qualités des enseignants}

La discipline forme avec le professeur un couple souvent indissociable dans l'esprit des élèves. Pariant sur sa solidité au cours du temps, nous avons cherché à révéler certains stéréotypes le concernant.

Cinq questions portaient sur les qualités souhaitées chez un enseignant selon la discipline enseignée. Les six réponses possibles ont été sélectionnées par une enquête préliminaire 
auprès d'élèves du primaire, du collège et du lycée. Le tableau 3 présente les résultats pour un échantillon de 7370 personnes.

Tableau 3: Les qualités pédagogiques souhaitées pour un proi esseur selon la matière qu'il enseigne Les cases encadrées en trait plein associent les qualites les plus citées par disciplin e. Les cases encadrées en pointilés associent les qualités les moins cilées par disciplin $\theta$.

\begin{tabular}{|c|c|c|c|c|c|c|}
\hline & $\begin{array}{c}1 \text { I sak } \\
\text { bien } \\
\text { expliquer. } \\
\text { avo ciar is }\end{array}$ & $\begin{array}{c}2 \text { If sait } \\
\text { sa taire } \\
\text { respecter das } \\
\text { blar os of } \\
\text { les respecie } \\
\text { auss: }\end{array}$ & 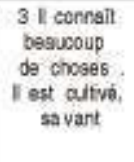 & $\begin{array}{c}\text { 4 II rend } \\
\text { sa } \\
\text { discipine } \\
\text { passiorrant o }\end{array}$ & $\begin{array}{c}5 \text { ll ast } \\
\text { sympatiqua } \\
\text { il a de } \\
\text { Thumour }\end{array}$ & $\begin{array}{l}6 \text { II es: } \\
\text { pesent at } \\
\text { répond als } \\
\text { quasions } \\
\text { des Élar as }\end{array}$ \\
\hline $\begin{array}{c}\text { Enseigne } \\
\text { la LS }\end{array}$ & $192 \%$ & $13 \beta \$$ & $13,0 \%$ & $33,1 \%$ & $122 \%$ & $87 \%$ \\
\hline $\begin{array}{c}\text { Ensoigne } \\
\text { les malhemmafcues }\end{array}$ & $35,3 \%$ & $7,7 \%$ & $3.5 \%$ & $17.2 \%$ & $7,3 \%$ & $290 \%$ \\
\hline $\begin{array}{l}\text { Enseigne } \\
\text { les sciences }\end{array}$ & $312 \%$ & $6,7 \%$ & $16,9 \%$ & $26,8 \%$ & $7,4 \%$ & $12,0 \%$ \\
\hline $\begin{array}{c}\text { Enseigne } \\
\text { Itrissoire-gtographie }\end{array}$ & $12,7 \%$ & $8,7 \%$ & $30,8 \%$ & $32.4 \%$ & $120 \%$ & $6.2 \%$ \\
\hline $\begin{array}{l}\text { Enseigne } \\
\text { leaducation phyaique }\end{array}$ & $6,7 \%$ & $31,6 \%$ & $14 \%$ & $14.5 \%$ & $413 \%$ & $4.5 \%$ \\
\hline
\end{tabular}
trop peu nombreux. Les résultats sont présentés au tableau 4.

Tableau 4 : Les deux qualités les plus souhaitées chez un prot esseur en fonction de sa dscipline et du pays de scolar isation.

\begin{tabular}{|c|c|c|c|}
\hline & D+A & $F$ & USA+G B \\
\hline LS & $4-1$ & $4-1$ & $4-1$ \\
\hline Mathematiques & $6-1$ & $1-6$ & $1-6$ \\
\hline Sciences & $1-4$ & $1-4$ & $1-4$ \\
\hline Histoire-Géographie & $3-1$ & $4-3$ & $4-3$ \\
\hline Education physique & $5-2$ & $5-2$ & $5-2$ \\
\hline
\end{tabular}


constate une grande homogénéité à l'exception des pays germaniques qui souhaitent que le professeur d'histoire et géographie soit clair plutôt que passionnant (caractères gras dans le tableau), ce qui coïncide avec la relation particulière qu'ils semblent entretenir avec l'histoire.

es stéréotypes transculturels s'explique par les caractéristiques de notre échantillon constitué par des lecteurs experts d'Harry Potter internautes, issus de "sociétés à valeurs rationnelles » où prédomine le modèle de l'école publique laïque. L'extension du sondage à d'autres types de communautés comme les "sociétés de type traditionnel », ou « les sociétés postmodernes » scandinaves ${ }^{5}$ donnerait certainement des résultats différents.

Il n'est pas surprenant que les effets des facteurs géopolitiques soient si présents dans une enquête sur l'éducation, vu la co-construction du politique et du scolaire qui caractérise les états modernes. Comme le dit J. -Cl. Ruano- Borbalan : «c'est l'école des Etats- Nations qui définit aujourd'hui dans la totalité des pays du monde, les fondements mémoriels majeurs (histoire nationale) et la définition de la culture légitime (littérature, humanisme, science, etc.)».

\section{L'identification des sujets aux disciplines}

Nous passons maintenant à l'analyse des réponses ${ }^{6}$ aux questions ouvertes « pourquoi ?» qui faisaient suite aux questions «quelle est votre discipline préférée? la moins aimée? ». Nous les avons soumises à une analyse de discours quantitative (le nombre d'items permettant le repérage de récurrences formelles), et qualitative (les marqueurs énonciatifs exprimant le lien du sujet à la discipline). Tout discours exprimant des préférences renseigne sur le "rapport à $»^{7}$, liaison stable entre un objet et un sujet. Analysable en composants mais perçu globalement, ce rapport s'exprime à travers un jugement d'identité. Celui-ci peut être obtenu par une question du type "pour vous les maths c'est quoi?». Ici, la stratégie de questionnement choisie étant indirecte, le jugement est apparu en présupposé : on aime la philosophie «car la philosophie c'est la vie », les mathématiques «parce que c'est logique ».

Comme tout jugement, le jugement d'identité renvoie à son énonciateur. Les justifications des préférences et des rejets des disciplines renseignent donc autant sur les apprenants que sur les disciplines.

28 Le descriptif des disciplines s'organise autour de leurs noms. Si ceux-ci font problème, cela peut provenir du référent - cas des Sciences Economiques et Sociales enseignées seulement depuis 1967- ou du signifié, dans la mesure où le découpage des savoirs fluctue en fonction des programmes, de l'avancée des connaissances et de leur transposition. L'onomastique est variée. On trouve des noms simples au singulier (technologie) et au pluriel (mathématiques), des noms composés (Physique- Chimie), et des expressions lexicalisées (Langues Vivantes, Sciences de la Terre et du Vivant). L'emploi fréquent du « et » trahit la faiblesse de la construction épistémique. Mais les noms simples sont presque plus trompeurs, car, loin de recouvrir une unité, ils sous-entendent des oppositions fondatrices, comme l'articulation langue - littérature en français.

En dégroupant les noms composés : «physique », «chimie » et non " physique- chimie», nous avons incité notre public à décomposer les signifiés des noms, passant du contenu

Tréma, 33 - 34 | 2011 
enseigné : la matière, à son investissement social dans et hors de l'école : la discipline. La construction du signifié peut être :

- - ignorée : « je me fiche sur quoi on vit et savoir toutes les choses que la SVT nous enseigne»;

- - paraphrasée, donc comprise, positivement : on aime la SVT « parce que c'est passionnant de connaître le fonctionnement de la planète et de l'être vivant », ou négativement :

" pourquoi étudier des petits cailloux ou des plantes sans intérêt »;

- - présupposée, donc dépassée, par le retour au référent social, celui des spécialités universitaires («j'adore [les Sciences de la Vie et de la Terre] sauf la bio- chimie », ou professionnelles : « je veux faire médecine ». pas!». la philosophie : disciplinaire.

Dans la description, les traits objectifs correspondent aux quatre aspects sous lesquels s'appréhende la discipline : programmes, enseignement, vie de classe, réflexion.

Les réformes des programmes font prendre conscience de l'historicité de la discipline. Ils relient l'histoire des sujets à celle de l'institution : «à l'époque, cette matière n'existait

Les situations didactiques sont évaluées en termes d'intérêt et d'efficacité : "ça pourrait être passionnant mais c'est mal enseigné »; la gestion de classe en termes de qualités humaines. La frontière entre les deux est poreuse, au moins pour les contre-indications des apprenants (phobies du sang pour les sciences, mauvaise image du corps pour l'EPS), et les comportements évaluatifs des enseignants («[en maths] il n’y a pas de discussion possible sur la note, alors que dans les matières littéraires, certains professeurs oublient de rester objectifs ». La réflexion est représentée par le jugement critique, ici à propos de

Je crois que l'exception française qui consiste en son enseignement en Terminales est une bonne chose, elle permet aux élèves de penser par eux-mêmes, cependant, je ne suis pas persuadée que l'enseignement en soit suffisamment « ludique » pour une matière que j'ai trouvé particulièrement difficile.

Les traits axiologiques indiquent les valeurs associées aux disciplines: rigueur (maths), ouverture (langues), réflexion (philo.). L'adhésion : « résoudons les problèmes avec notre esprit si possible pas avec les armes ", prouve l'affiliation de l'apprenant à la discipline comme groupe de référence, ici la communauté idéale des philosophes. Inversement, le rejet « qui suis-je, on s'en fout ! » renvoie au groupe d'appartenance subi qu'est la classe.

L'intégration de la discipline dans le schéma de soi passe par ${ }^{8}$ :

- - l'auto- valorisation : « ma meilleure matière »;

- - la fidélité à soi : «j'y ai réussi et j'en ai fait mon métier »;

- - l'autoévaluation, qui permet de se comparer aux autres : «j'y ai les meilleures notes»;

- - l'amélioration de soi : « le français me fait progresser d'année en année ».

Ces facteurs provoquent l'apparition du discours expert: « je trouve les démonstrations passionnantes, à partir de quelques hypothèses on arrive parfois à des conclusions très étonnantes. Et puis la magie des nombres imaginaires rhalala", et celle de l'autodéfinition. Celle-ci est soit disciplinaire: "je suis en licence d'histoire ", soit professionnelle : « je suis historien », quand le métier est venu confirmer la préférence 


\section{Les stéréotypes disciplinaires} questions sur les profils d'enseignants forcent les lecteurs à objectiver les stéréotypes disciplinaires. La demande de justification des choix les incite au contraire à les intérioriser. Sémantiquement, le stéréotypage est une généralisation ${ }^{9}$ qui relève plus de l'abduction (à partir de vérités d'opinion) que de la déduction (à partir de vérités démontrées). Il s'exprime à travers des figures de pensée comme la comparaison (on aime les SVT parce que " c'est du concret, pas comme les maths ", mais surtout des figures de mots ${ }^{10}$ basées sur des rapprochements intuitifs.

La synecdoque généralisante réduit la discipline à un incident marquant : la chimie, c'est "pour faire exploser le labo" tandis que la synecdoque particularisante l'étend démesurément: "la physique -chimie recouvre toutes les sciences». La syllepse de synecdoque l'enferme dans une tautologie mystique: «pourquoi les mathématiques? Parce que ce sont les mathématiques justement». La personnification : «[j'aime les mathématiques] parce qu'ils (sic) deviennent intéressants lorsqu'on s'intéressent à eux » débouche logiquement sur l'assimilation, les maths devenant une « une seconde nature ».

La stéréotypie véhiculée par le discours autobiographique est marquée par le rapport désirant de l'apprenant à la discipline. En même temps, on s'aperçoit que le stéréotypage est producteur de méta- savoirs qui ne sont ni faux a priori ni anodins. C'est d'ailleurs ce qui a contribué à la réhabilitation du stéréotype dans les sciences du discours et la didactique depuis une décennie.

Le stéréotype a une incidence sur la progression didactique, non seulement parce qu'il fait partie intégrante de la vision du monde des apprenants, mais aussi parce qu'il peut être perçu de trois façons :

- - naïve, «j'aime les maths à cause de la logique implacable des mathématiques », position en je centrée sur le sujet ;

- - critique, «bizarrement, oui, j'adore les mathématiques » position en tu, qui prend en compte le point de vue adverse ;

- - réhabilitatrice ${ }^{11}$, « c'est logique, c'est carré, et ce n'est absolument pas abstrait », position en il recentrée sur l'objet.

Déterminer la posture de réception du stéréotype est donc un préalable à son exploitation.

La réception du stéréotype est à la fois un acte de discours et une prise de position sociale. «Bizarrement, oui, j'adore les mathématiques » est un contre- argument dans un débat imaginaire. En remettant en cause un stéréotype négatif dominant, l'énonciateur s'attend à être lui-même jugé négativement. Chaque groupe disciplinaire est producteur d'hétérostéréotypes qui lui servent à désigner les autres disciplines, et d'autostéréotypes auxquels il s'identifie. La coprésence des disciplines dans l'école suffit d'ordinaire à limiter la bipolarisation. D'ailleurs certains sondés ont refusé de choisir entre elles. Mais elle ressurgit chaque fois que l'apprenant se sent atteint dans son identité personnelle par les décisions de l'institution.

41 La présence du stéréotype oppositif scientifique/littéraire dans l'échantillon français est liée aux modes d'évaluation et d'orientation en France. Radicalisée - «littéraire» devenant synonyme de "non scientifique » et vice-versa -, l'opposition efface les 
spécificités disciplinaires jusqu'à compromettre l'identité autostéréotypique : «j'ai beau être scientifique je n'y ai jamais rien compris [à la physique] ». En retour, elle permet de justifier l'échec par la prédestination («trop abstrait pour moi [les maths] !!! suis une littéraire »; «trop littéraire[la philo]»); ou de rejeter la responsabilité sur le groupe dominant ( je me sens rejetée par un monde qui absorbe les filières scientifiques et rejette les littéraires »). L'interlocuteur exposé à l'hétérostéréotype a le choix entre le subir («trop littéraire, m’a-t-on dit!»), le retourner («c'est trop compliqué pour mon petit cerveau littéraire ») ou l'assumer ( «je ne suis pas une scientifique mais plus une littéraire »), bâtissant alors son identité scolaire par défaut.Les savoirs scolaires sont à la fois déterminés par les cultures nationales et linguistiques, et relativement indépendants. Il existe une méta- culture scolaire mondiale sur laquelle on peut s'appuyer pour gérer la circulation des apprenants et des savoirs.

Le rapport de l'apprenant aux savoirs est orienté par sa relation d'une part au groupe classe et à la « matière » imposée par l'institution, d'autre part au groupe disciplinaire en tant que communauté d'experts productrice de savoir.

Les stéréotypes disciplinaires jouent un rôle prépondérant dans la construction de l'identité scolaire. Descriptifs, ils servent de repères dans la cartographie des savoirs. Axiologiques, ils situent les disciplines dans le projet éducatif. Le stéréotypage du groupe disciplinaire permet à l'apprenant d'inscrire dans son image sociale son expertise ou son ignorance, au prix de renoncements et de simplifications dommageables autant pour les personnes que pour les savoirs. Les positions de l'amateur et de l'autodidacte, aux confins du groupe de référence et du groupe d'appartenance, apparaissent comme des tentatives pour négocier une place honorable entre rejet institutionnel et liberté de choix.

Il est donc indispensable de repérer les stéréotypes disciplinaires et interdisciplinaires pour les faire évoluer dans un sens favorable aux apprentissages. 


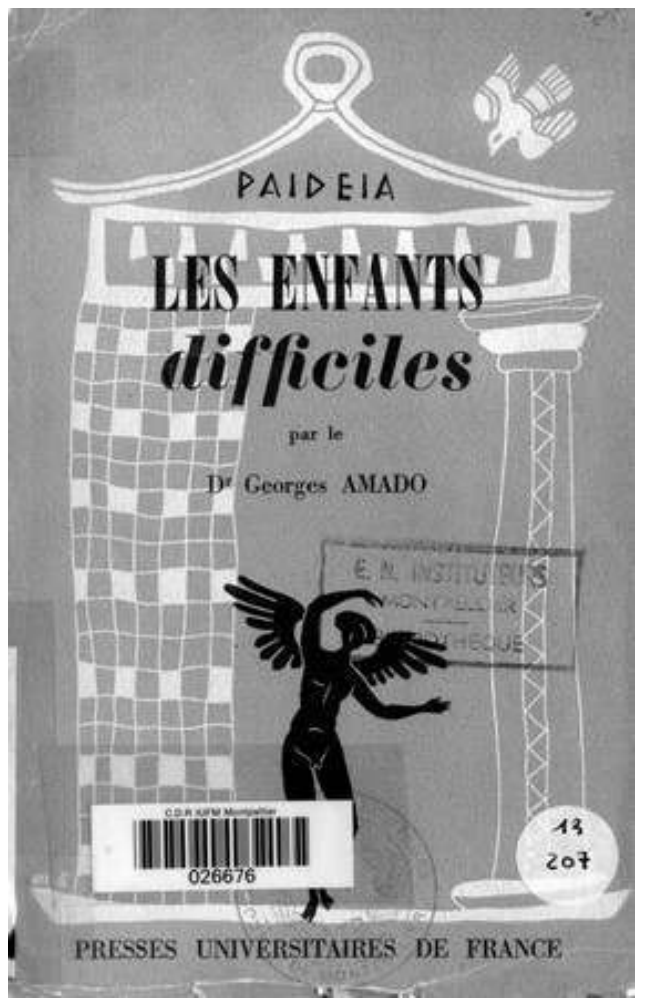

IMAgE tiRÉE DE Les enfants difficiles, $3^{e}$ section : psychiatrie infantine / DR GEORgeS AMADO. PARIS : PRESSE UNIVERSITAIRE DE FRANCE, 1955. (COLL. PAIDEIA). 180P. : COUV. ILL. ; 19CM - COUVERTURE. SOURCE : CEDRHE.

\section{NOTES}

1. Rappelons avec Claude THELOT que « la position du père signifie plus qu'elle-même : en raison notamment de l'importance de l'homogamie, elle mesure ou synthétise [...] le milieu social d'origine », (THELOT, C. (1988), Tel père, tel fils ? Paris : Hachette,Pluriel, 17

2. COMTE, A. (1850) Cours de philosophie positive T. 1, Paris : Editions Bachelier, 57

3. PINEAU C. (1989) Revue en ligne EPS n²11-216, 25

4. 1989 , ibid

5. RUANO-BORBALAN, J.-Cl. (2004) «Culture, valeurs: tous post-modernes », Identité(s), (Halpern, Ruano-Borbalan eds) Editions Science Humaines, 269

6. «Introduction générale : la construction de l'identité », ibid., 9

7. Limitées ici au groupe LS français, soit 3252 justifications pour matière préférée et 3311 pour matière la moins aimée

8. Cf. Pour une clinique du rapport au savoir (1996) BEILLEROT, J., BLANCHARD-LAVILLE, Cl., MOSCONI, N. (sous la direction de) Paris : L'Harmattan

9. cf. MARTINOT, D. (2004) « Le soi en psychologie sociale », Identité(s), 42

10. Cf. AMOSSY, R. et HERSCHBERG Pierrot, A. (1997) Stéréotypes et clichés, Paris : Nathan, 50

11. Cf. FONTANIER, P. (1977) Les Figures du discours, Paris : Flammarion 


\section{RÉSUMÉS}

Les disciplines faisant le lien entre le monde du travail et les apprentissages scolaires, leur gestion dans l'institution scolaire répercute l'image sociale des professions qui leur sont associées et contribue à sa reproduction. Une enquête internationale sur les représentations scolaires des lecteurs d'Harry Potter (6 langues, plus de 9000 réponses) nous a permis d'étudier : 1) la répartition des identifications disciplinaires selon l'âge, le sexe, le niveau d'études, la situation familiale, la langue, le pays 2) le système énonciatif et argumentatif qui nourrit le jugement d'identité disciplinaire («je / il suis /est matheux», «je/il suis/est littéraire ») durant la scolarité et au-delà. Notre étude montre : 1) le rôle bloquant des stéréotypes disciplinaires auto et hétéro - appliqués, 2) la nécessité de les repérer, au niveau national comme international, pour les faire évoluer dans un sens favorable aux apprentissages et à la construction de l'identité apprenante.

This article describes the principles and concrete outcomes of a project entitled CONBAT+ (Content-Based teaching + plurilingualism). This project is being held within the 2008-2011 medium programme of the European Centre for Modern Languages (ecml- Graz, Council of Europe). The aim of the project is to enrich the CLIL approach with a plurilingual and pluricultural dimension. The teaching units produced in this programme are based on a conception of teaching and learning at school as a collaborative construction of knowledge in a social context and on a conception of languages as social modes of acting and vectors of otherness and diversity. They aim at developing the construction of the students' identities. The concept of identity is here considered as complex (in the sense used by Morin, 1990) and is analysed through an integrative approach of language and languages.

\section{INDEX}

Mots-clés : autobiographie, discipline, enquête, identité, jugement

\section{AUTEURS}

\section{NICOLE BIAGIOLI}

IUFM de l'académie de Nice - Université Nice Sophia Antipolis

\section{JEAN-JACQUES LEGENDRE}

IUFM de l'académie de Nice - Université Nice Sophia Antipolis 